\title{
Vacuum Stability Constraints in Models with Extended Scalar Sectors
}

\section{Jonas Wittbrodt*}

DESY, Notkestrasse 85, 22607 Hamburg, Germany

E-mail: Jonas.Wittbrodtedesy.de

\begin{abstract}
We discuss constraints from the stability of the electroweak vacuum in BSM models with extended scalar sectors. We present an efficient and reliable method to obtain vacuum stability constraints at tree-level based on the straight path approximation for the bounce action. In the MSSM, we obtain vacuum stability constraints on a benchmark scenario that are complementary to experimental limits and illustrate the reliability of our method. In a parameter scan of the type I N2HDM, we show a direct constraint from vacuum stability on the possible values for the diphoton rate of the $125 \mathrm{GeV}$ Higgs boson.
\end{abstract}

ALPS 2019 An Alpine LHC Physics Summit

April 22 - 27, 2019

Obergurg, Austria

${ }^{*}$ Speaker. 


\section{Vacuum Stability in Extended Scalar Sectors}

Sufficient stability of the electroweak (EW) vacuum throughout the age of the universe is a necessary condition for any model that aims to describe nature. In the SM this is trivially fulfilled at the EW, scale as the EW vacuum is the only minimum of the scalar potential. Even when extrapolating the SM to extremely high energies, the vacuum remains sufficiently long-lived with the latest calculations obtaining lifetimes around $\sim 10^{140}$ years [1]. Beyond the SM (BSM) theories with extended scalar sectors can behave very differently. In this case, multiple physically distinct minima of the scalar potential can be present already at tree-level and at the EW scale. The following discussion of vacuum stability in such models is based on refs. [2, 3].

Any renormalizable tree-level scalar potential of $n$ real scalar fields $\phi_{a}$ can be expanded into the form

$$
V(\vec{\phi})=\lambda_{a b c d} \phi_{a} \phi_{b} \phi_{c} \phi_{d}+A_{a b c} \phi_{a} \phi_{b} \phi_{c}+m_{a b}^{2} \phi_{a} \phi_{b}+t_{a} \phi_{a}+c,
$$

with real coefficient tensors containing all possible terms of non-negative mass dimension. Expanding around the electroweak vacuum, $\vec{\varphi}=\vec{\phi}-\vec{\phi}_{v}$, and introducing polar coordinates $\vec{\varphi} \rightarrow \varphi \hat{\varphi}$ with an appropriately normalized unit vector $\hat{\varphi}$ (see ref.[2]) leads to

$$
V(\varphi, \hat{\varphi})=\lambda(\hat{\varphi}) \varphi^{4}-A(\hat{\varphi}) \varphi^{3}+m^{2}(\hat{\varphi}) \varphi^{2} .
$$

This potential along a specific direction $\hat{\varphi}$ starting from the EW vacuum is given by a quartic polynomial with a minimum at the origin. Requiring the EW vacuum to be a minimum of a bounded scalar potential leads to $m^{2}(\hat{\varphi}), \lambda(\hat{\varphi})>0 \forall \hat{\varphi}$ and $A(\hat{\varphi})>0$ can be chosen without loss of generality through $\varphi \rightarrow-\varphi$ and $A(\hat{\varphi}) \rightarrow-A(\hat{\varphi})$. In the left panel of fig. 1, we show the different possibilities for the shape of the potential, eq. (1.2). Along a fixed direction $\hat{\varphi}$ there can be at most one additional minimum, which is deeper than the EW vacuum at the origin if

$$
A(\hat{\varphi})^{2}>4 m^{2}(\hat{\varphi}) \lambda(\hat{\varphi})
$$

The decay rate of a vacuum state $\Gamma$ normalized to the spacial volume $V_{S}$ is given by [4]

$$
\frac{\Gamma}{V_{S}}=K \mathrm{e}^{-B}
$$

The bounce action $B$ is the dominant contribution while the prefactor $K$ is commonly estimated as $K \sim \Lambda^{4}$ on dimensional grounds, where $\Lambda$ is the scale of the theory. Assuming that vacuum decay happens along a straight line described by eq. (1.2) allows an approximate calculation of $B$ using a semianalytical result for tunnelling in a polynomial potential [5]. If there are several minima that the EW vacuum can tunnel into, we call the one with the smallest value of $B$ the most dangerous minimum. Figure 1 (left) shows the lifetime of the EW vacuum normalized to the age of the universe as a function of $\Lambda$ and $B$. The lines indicate where decay or survival throughout the age of the universe is expected at $5 \sigma$. These boundaries between stability and instability traverse the range $390<B<440$ when varying $\lambda$ from $1 \mathrm{GeV}$ to $100 \mathrm{TeV}$. Since the dependence of the lifetime on the value of $\Lambda$ is so mild, we choose to judge the stability solely based on the value of $B$ and treat $390<B<440$ as an uncertainty band due to the unknown $K$. 

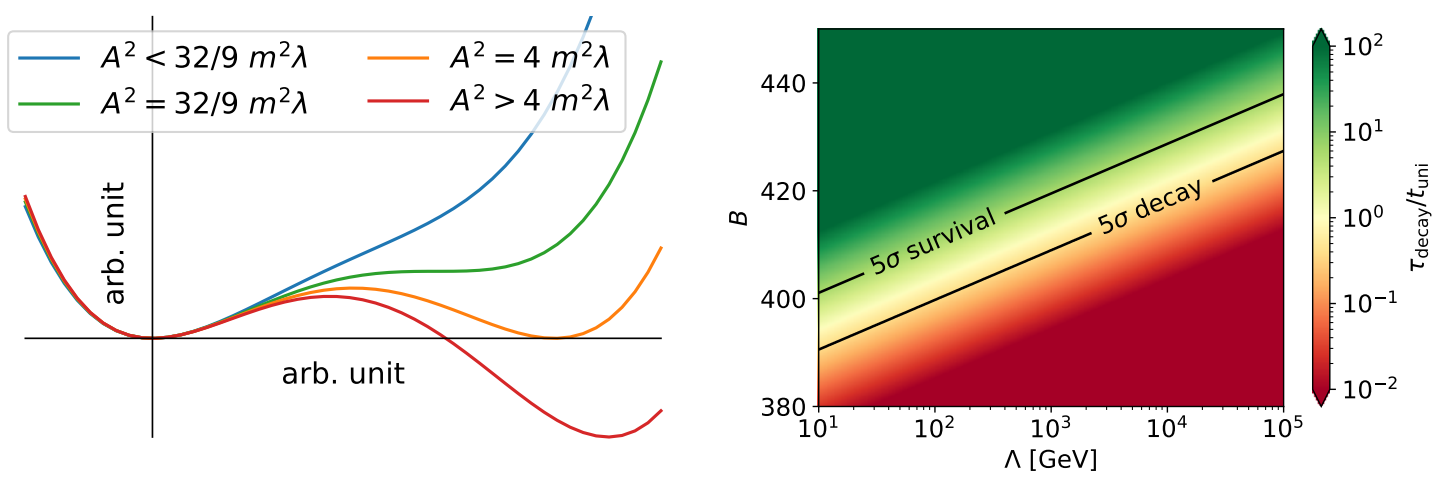

Figure 1: The left panel shows the possible configurations of eq. (1.2) depending on the values of the parameters for a given $\hat{\varphi}$. The right panel shows the lifetime of the EW vacuum normalized to the age of the universe as a function of $B$ and $\Lambda$ of eq. (1.4). The contours indicate expected survival and expected decay at $5 \sigma$.

\subsection{Vacuum Stability Constraints}

We distinguish the following categories of stability for the EW vacuum based on the value of $B$ for tunnelling to the most dangerous minimum:

- absolutely stable, the EW vacuum is the global minimum of the scalar potential;

- long-lived, metastable, the fastest decay mode of the EW vacuum has $B>440$;

- short-lived, unstable, the fastest decay mode of the EW vacuum has $B<390$.

The intermediate region, $390<B<440$, accounts for the uncertainty from the unknown $K$ as discussed above. Using the tree-level scalar potential and the straight path approximation is a highly efficient method to obtain vacuum stability constraints that is usable even in large BSM parameter scans.

In supersymmetric theories, vacuum stability constraints are highly interesting due to the large number of scalar fields. The left panel of fig. 2 shows vacuum stability constraints in the $M_{h}^{125}(\tilde{\tau})$ benchmark scenario [6] of the MSSM. The majority of the parameter plane has either long-lived or absolutely stable EW vacua and is unconstrained by vacuum stability. However, at high $\tan \beta$, the EW vacuum is short-lived, and the red region is excluded by vacuum stability. Also note that the yellow band indicating the uncertainty from the estimation of $K$ is thin, showing that the precise value used for the boundary between long- and short-lived vacua has little impact on the allowed parameter space. In the parameter region where both $\tan \beta$ and $m_{A}$ are large, the vacuum stability constraint is complementary to experimental limits on the benchmark scenario. See ref. [2] for a more detailed discussion.

Figure 2 (right) shows the impact of the charged Higgs boson $H^{ \pm}$on the signal strength $\mu_{\gamma \gamma}$ of $p p \rightarrow h_{125} \rightarrow \gamma \gamma$ in the type I N2HDM [7]. Without vacuum stability constraints, substantial enhancements are possible for low to medium $m_{H^{ \pm}}$as a result of charged Higgs contribution to the loop induced decay. However, most of these parameter points have unstable EW vacua making enhancements of $\mu_{\gamma \gamma}$ above $\sim 1.03$ only allowed for very low $m_{H^{ \pm}} \lesssim 400 \mathrm{GeV}$. This is an example of vacuum stability constraints directly impacting the phenomenological predictions of a model. 

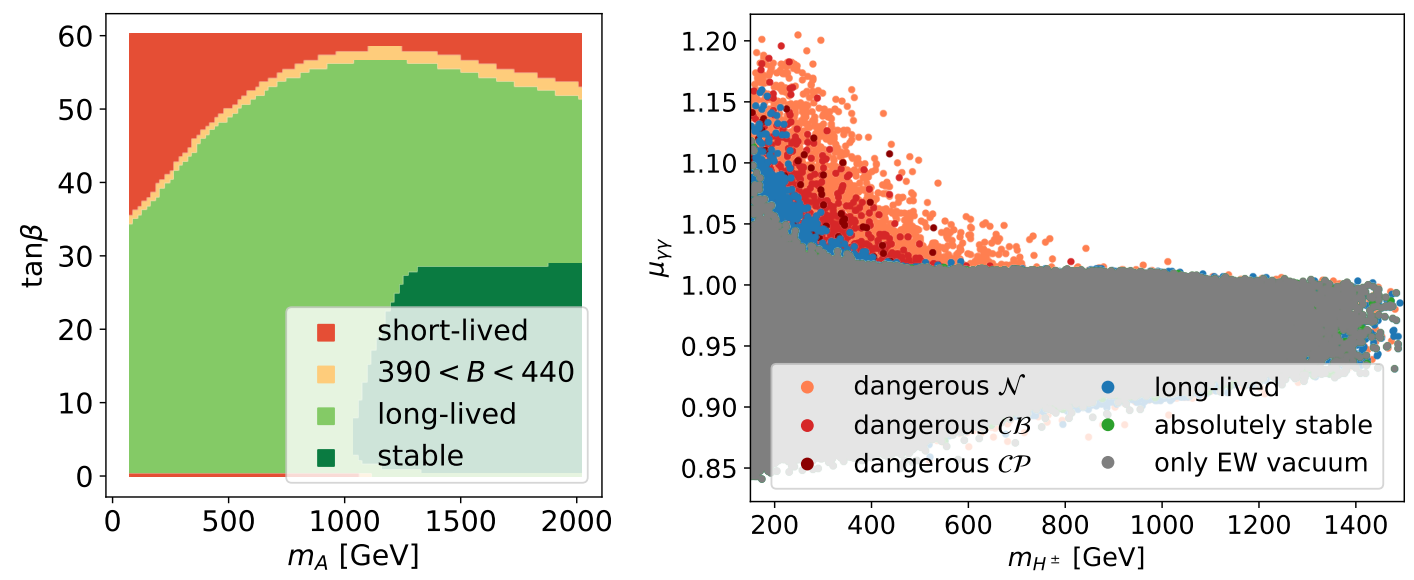

Figure 2: Vacuum stability constraints on the MSSM benchmark scenario $M_{h}^{125}(\tilde{\tau})$ [6] in the $m_{A}-\tan \beta$ plane (left). Stability of the EW vacuum in a parameter scan of the N2HDM in the plane of the signal rate observable $\mu_{\gamma \gamma}$ and the charged Higgs mass $m_{H^{ \pm}}$. More stable points are plotted on top and dangerous points denote different kinds of minima into which short-lived tunnelling of the EW vacuum can occur.

In this case, vacuum stability constrains the value of the $h_{125} H^{+} H^{-}$coupling and thus limits the possible enhancement of $\Gamma\left(h_{125} \rightarrow \gamma \gamma\right)$. The vacuum structure of the N2HDM is discussed in more detail in ref. [3]

\subsection{Summary}

We have discussed vacuum stability constraints in BSM models with extended scalar sectors. We have presented an approximate method based on the straight path approximation that allows an efficient and reliable evaluation for use in large parameter scans. Results obtained with this method in the MSSM and the N2HDM have illustrated the relevance of vacuum stability constraints in phenomenological studies and their possible complementarity to experimental results.

\section{References}

[1] A. Andreassen, W. Frost, M. D. Schwartz, Phys. Rev. D97 (2018) 056006 [1707.08124]

S. Chigusa, T. Moroi, Y. Shoji, Phys. Rev. Lett. 119 (2017) 211801 [1707.09301], Phys. Rev. D97 (2018) 116012 [1803.03902]

[2] W. G. Hollik, G. Weiglein, J. Wittbrodt, JHEP 03 (2019) 109 [1812.04644]

[3] P. M. Ferreira, R. Santos, M. Mühlleitner, G. Weiglein, J. Wittbrodt, (2019), [1905.10234]

[4] S. R. Coleman, Phys. Rev. D15 (1977) 2929

C. G. Callan Jr., S. R. Coleman, Phys. Rev. D16 (1977) 1762

[5] F. C. Adams, Phys. Rev. D48 (1993) 2800, [hep-ph/9302321]

[6] E. Bagnaschi et.al., Eur. Phys. J. C79 (2019) 617, [1808.07542]

[7] M. Mühlleitner, M. O. P. Sampaio, R. Santos, J. Wittbrodt, JHEP 03 (2017) 094, [1612.01309] 